\title{
SOME RESULTS ON COMPACT ALMOST RICCI SOLITONS WITH NULL COTTON TENSOR
}

\author{
A. BARROS AND I. EVANGELISTA
}

\begin{abstract}
The aim of this paper is to prove that a compact almost Ricci soliton with null Cotton tensor is isometric to a standard sphere provided one of the following conditions associated to the Schouten tensor holds: the second symmetric function is constant and positive; two consecutive symmetric functions are non null multiple or some symmetric function is constant and the quoted tensor is positive.
\end{abstract}

\section{Introduction}

The concept of almost Ricci soliton was introduced by Pigola et al. in [15], where essentially they modified the definition of a Ricci soliton by permitting to the parameter $\lambda$ to be a variable function. More precisely, we say that a Riemannian manifold $\left(M^{n}, g\right)$ is an almost Ricci soliton if there exist a complete vector field $X$ and a smooth soliton function $\lambda: M^{n} \rightarrow \mathbb{R}$ satisfying

$$
\operatorname{Ric}+\frac{1}{2} \mathcal{L}_{X} g=\lambda g,
$$

where Ric and $\mathcal{L}$ stand for the Ricci curvature tensor and the Lie derivative, respectively. We shall refer to this equation as the fundamental equation of an almost Ricci soliton $\left(M^{n}, g, X, \lambda\right)$. We say that an almost Ricci soliton is shrinking, steady or expanding provided $\lambda>0, \lambda=0$ or $\lambda<0$, respectively, otherwise we say that it is indefinite. When $X=\nabla f$ for some smooth function $f$ on $M^{n}$, we say that it is a gradient almost Ricci soliton. In this case, identity (1.1) becomes

$$
\operatorname{Ric}+\nabla^{2} f=\lambda g
$$

Received April 16, 2016; received in final form December 29, 2016.

Partially supported by CNPq - Brazil.

2010 Mathematics Subject Classification. Primary 53C25, 53C20, 53C21. Secondary 53C24. 
where $\nabla^{2} f$ stands for the Hessian of $f$. Further, an almost Ricci soliton is trivial provided $X$ is a Killing vector field, otherwise it will be called a nontrivial almost Ricci soliton. We point out that when $X$ is a Killing vector field and $n \geq 3$, we have that $M$ is an Einstein manifold since Schur's lemma ensures that $\lambda$ is constant.

We highlight that Ricci solitons also correspond to self-similar solutions of Hamilton's Ricci flow, for more details about Ricci soliton see, for example, [8]. In this perspective, Brozos-Vázquez, García-Río and Valle-Regueiro [6] observed that some proper gradient Ricci almost solitons correspond to selfsimilar solutions of the Ricci-Bourguignon flow, which is a geometric flow given by

$$
\frac{\partial}{\partial t} g(t)=-2(\operatorname{Ric}(t)-k R(t) g(t))
$$

where $k \in \mathbb{R}$ and $R$ stands for the scalar curvature. This flow can be seen as an interpolation between the flows of Ricci and Yamabe. For more details on Ricci-Bourguignon flow, we recommend [9].

It is important to emphasize that the round sphere does not admit a (nontrivial) Ricci soliton structure. However, Barros and Ribeiro Jr [3] showed an explicit example of an almost Ricci soliton on the standard sphere. For this reason, it is interesting to know if, in the compact case, this is the unique example with non-constant soliton function $\lambda$. In this sense, Barros and Ribeiro Jr [3] proved that a compact gradient almost Ricci soliton with constant scalar curvature must be isometric to a standard sphere. Afterward, Barros, Batista and Ribeiro Jr [2] proved that every compact almost Ricci soliton with constant scalar curvature is gradient. In [5], Costa, Brasil and Ribeiro Jr showed that under a suitable integral condition, a 4-dimensional compact almost Ricci soliton is isometric to the standard sphere $\mathbb{S}^{4}$. While Ghosh [10] was able to prove that if a compact $K$-contact metric is a gradient almost Ricci soliton, then it is isometric to a unit sphere. We recall that Barros, Batista and Ribeiro Jr [1] proved that under a suitable integral condition a locally conformally flat compact almost Ricci soliton is isometric to a standard sphere $\mathbb{S}^{n}$. For more details see, for instance, [2], [1], [10], [13] and [16].

When $M$ is a compact manifold the Hodge-de Rham decomposition theorem (see for instance [17]) asserts that $X$ can be decomposed as a sum of a gradient of a function $h$ and a divergence-free vector field $Y$, that is,

$$
X=\nabla h+Y
$$

where $\operatorname{div} Y=0$. From now on, we consider $h$ the function given by this decomposition.

Henceforth, we denote by $M^{n}, n \geq 3$, a compact connected oriented manifold without boundary. Now we remember some basic facts about symmetric 
functions. Let $A$ be the Schouten tensor and $\sigma_{k}(A)$ be the symmetric functions associated to $A$ defined as follows

$$
\operatorname{det}(I+t A)=\sum_{k=0}^{n} \sigma_{k}(A) t^{k} .
$$

Since $A$ is symmetric, then $\left(\begin{array}{l}n \\ k\end{array}\right) S_{k}(A)=\sigma_{k}(A)$ coincides with the $k$ th elementary symmetric polynomial of the eigenvalues $\lambda_{i}(A)$ of $A$, that is,

$$
\sigma_{k}(A)=\sigma\left(\lambda_{1}(A), \ldots, \lambda_{n}(A)\right)=\sum_{i_{1}<\cdots<i_{k}} \lambda_{i_{1}}(A) \cdots \lambda_{i_{k}}(A),
$$

for more details about symmetric functions see, for instance, [12]. It should be emphasized that the assumption of constant scalar curvature is equivalent to require that the trace of the Schouten tensor is constant. Indeed, if we denote by $A$ the Schouten tensor, then $\operatorname{tr} A=\frac{1}{2} \frac{n-2}{(n-1)} R$, where $R$ stands for the scalar curvature of $M$. Since $n S_{1}(A)=\operatorname{tr} A$, this is in turn equivalent to require that the first symmetric function of $A$ is constant. Inspired by the historical development on the study of compact almost Ricci soliton. In this paper, we investigate which geometric implication has the assumption that the second symmetric function $S_{2}$ associated to the Schouten tensor is constant and positive on a compact almost Ricci soliton. More precisely, we have the following result.

THEOREM 1. Let $\left(M^{n}, g, X, \lambda\right)$ be a non-trivial compact oriented almost Ricci soliton such that the Cotton tensor is identically zero. Then, $M^{n}$ is isometric to a standard sphere $\mathbb{S}^{n}$ provided that one of the next condition is satisfied:

(1) $S_{2}(A)$ is constant and positive.

(2) $S_{k}(A)$ is nowhere zero on $M$ and $S_{k+1}(A)=c S_{k}(A)$, where $c \in \mathbb{R} \backslash\{0\}$, for some $k=1, \ldots, n-1$.

(3) Ric $\geq \frac{R}{n} g$, with $R>0$, and $\int_{M} S_{k}(A) \Delta h \geq 0$ for some $2 \leq k \leq n-1$.

(4) $S_{k}(A)$ is constant for some $k=2, \ldots, n-1$, and $A>0$.

We highlight that the symmetric functions associated to the Schouten tensor were used by $\mathrm{Hu}, \mathrm{Li}$ and Simon [12] to study locally conformally flat manifolds. By assuming that the Weyl tensor vanishes, then the conclusion of item (4) in Theorem 1 follows directly from Theorem 1 obtained in [12]. In this direction, we point out that item (1) and item (4) of Theorem 1 improve Theorem 1 in [12] for compact almost Ricci solitons under the hypothesis of Cotton tensor identically zero.

The paper is organized as follows: in Section 2, we present some basic notations and definitions; Section 2.2 is devoted to define Newton transformations associated to a symmetric $(0,2)$ tensor and to compute the divergence of such transformations whereas in Section 2.3 we establish some integral formulae for compact oriented almost Ricci soliton associated to the Schouten tensor. 
Finally, in Section 3, we prove our main result as an application of the integral formulae obtained in the previous section.

\section{Preliminaries}

2.1. Notations. Let $\left(M^{n}, g\right)$ be a smooth, $n$-dimensional Riemannian manifold with metric $g$. We denote by $\operatorname{Rm}(X, Y) Z$ the Riemann curvature operator defined as follows

$$
\operatorname{Rm}(X, Y) Z=\nabla_{X} \nabla_{Y} Z-\nabla_{Y} \nabla_{X} Z-\nabla_{[X, Y]} Z
$$

and we also denote by $\operatorname{Ric}(X, Y)=\operatorname{tr}(Z \rightarrow \operatorname{Rm}(Z, X) Y)$ the Ricci tensor, and $R=\operatorname{tr}(\mathrm{Ric})$ the scalar curvature. We have the well-known formula

$$
(\operatorname{div} \operatorname{Rm})(X, Y, Z)=\nabla_{X} \operatorname{Ric}(Y, Z)-\nabla_{Y} \operatorname{Ric}(X, Z) .
$$

Let $A=\operatorname{Ric}-\frac{R}{2(n-1)} g$ denote the Schouten tensor, which is a $(0,2)$ symmetric tensor. The Weyl tensor is given by

$$
\mathrm{Rm}=W+\frac{1}{n-2}(A \odot g),
$$

where $\odot$ means the Kulkarni-Nomizu product defined by the following formula

$$
(\alpha \odot \beta)_{i j k l}=\alpha_{i l} \beta_{j k}+\alpha_{j k} \beta_{i l}-\alpha_{i k} \beta_{j l}-\alpha_{j l} \beta_{i k},
$$

where $\alpha, \beta$ are $(0,2)$ tensor. Finally we define the Cotton tensor as follows

$$
C_{i j k}=\nabla_{i} A_{j k}-\nabla_{j} A_{i k}
$$

It is well known that

$$
\nabla^{l} W_{i j k l}=\frac{n-3}{n-2} C_{i j k} .
$$

From identities (2.4) and (2.5), we see that for $n \geq 4$ if the Weyl tensor vanishes, then the Cotton tensor also vanishes. We also see that when $n=3$ the Weyl tensor always vanishes, but the Cotton tensor does not vanish in general. We say that a manifold has harmonic Weyl tensor provided that $\operatorname{div} W=0$, where div means the divergence of the tensor. By (2.5) we also have that for $n \geq 4$, the Cotton tensor is identically zero if and only if the Weyl tensor is harmonic.

2.2. Newton transformations. Let $T$ be a symmetric $(0,2)$ tensor and $\sigma_{k}(T)$ be the symmetric functions associated to $T$ defined as follows

$$
\operatorname{det}(I+s T)=\sum_{k=0}^{n} \sigma_{k}(T) s^{k},
$$


where $\sigma_{0}=1$. Since $T$ is symmetric, then $\left(\begin{array}{l}n \\ k\end{array}\right) S_{k}(T)=\sigma_{k}(T)$ coincides with the $k$ th elementary symmetric polynomial of the eigenvalues $\lambda_{i}(T)$ of $T$, that is,

$$
\sigma_{k}(T)=\sigma\left(\lambda_{1}(T), \ldots, \lambda_{n}(T)\right)=\sum_{i_{1}<\cdots<i_{k}} \lambda_{i_{1}}(T) \cdots \lambda_{i_{k}}(T), \quad 1 \leq k \leq n .
$$

For simplicity, we do not distinguish between the $(0,2)$ tensor $T$ and the operator $\widetilde{T}: \mathfrak{X}(M) \rightarrow \mathfrak{X}(M)$, that is a $(1,1)$ tensor, such that $T(X, Y)=\langle\widetilde{T} X, Y\rangle$. Introduce the Newton transformations $P_{k}(T): \mathfrak{X}(M) \rightarrow \mathfrak{X}(M)$, arising from the operator $T$, by the following inductive law

$$
P_{0}(T)=I, \quad P_{k}(T)=\left(\begin{array}{l}
n \\
k
\end{array}\right) S_{k}(T) I-T P_{k-1}(T), \quad 1 \leq k \leq n
$$

or, equivalently,

$$
\begin{aligned}
P_{k}(T)= & \left(\begin{array}{l}
n \\
k
\end{array}\right) S_{k}(T) I-\left(\begin{array}{c}
n \\
k-1
\end{array}\right) S_{k-1}(T) T+\cdots \\
& +(-1)^{k-1}\left(\begin{array}{c}
n \\
1
\end{array}\right) S_{1}(T) T^{k-1}+(-1)^{k} T^{k} .
\end{aligned}
$$

Using the Cayley-Hamilton theorem, we get $P_{n}(T)=0$.

Note that $P_{k}(T)$ is a self-adjoint operator that commutes with $T$ for any $k$. Furthermore, if $\left\{e_{1}, \ldots, e_{n}\right\}$ is an orthonormal frame on $T_{p} M$ diagonalizing $T$, then

$$
\left(P_{k}(T)\right)_{p}\left(e_{i}\right)=\mu_{i, k}(T)_{p} e_{i},
$$

where

$$
\mu_{i, k}(T)=\sum_{i_{1}<\cdots<i_{k}, i_{j} \neq i} \lambda_{i_{1}}(T) \cdots \lambda_{i_{k}}(T)=\frac{\partial \sigma_{k+1}}{\partial x_{i}}\left(\lambda_{1}(T), \ldots, \lambda_{n}(T)\right) .
$$

Moreover, we have the well-known formulae

$$
\left\{\begin{array}{l}
\operatorname{tr}\left(T P_{k}(T)\right)=c_{k} S_{k+1}(T), \\
\operatorname{tr}\left(P_{k}(T)\right)=c_{k} S_{k}(T)
\end{array}\right.
$$

where

$$
c_{k}=(n-k)\left(\begin{array}{l}
n \\
k
\end{array}\right)=(k+1)\left(\begin{array}{c}
n \\
k+1
\end{array}\right) .
$$

The divergence of $P_{k}(T)$ is defined as follows

$$
\operatorname{div} P_{k}(T)=\operatorname{tr}\left(\nabla P_{k}(T)\right)=\sum_{i=1}^{n} \nabla_{e_{i}} P_{k}(T)\left(e_{i}\right),
$$


where $\left\{e_{1}, \ldots, e_{n}\right\}$ is a local orthonormal frame on $M$. Our aim is to compute the divergence of $P_{k}(T)$. The following definition is important in the sequel. Introduce the tensor $D$ by

$$
D_{i j k}=\nabla_{i} T_{j k}-\nabla_{j} T_{i k}
$$

Note that when $T$ is the Ricci tensor, then by equation (2.1) $D=\operatorname{div} R \mathrm{Rm}$, and when $T$ is the Schouten tensor, then $D$ is just the Cotton tensor.

Lemma 1. Let $P_{k}(T)$ be the Newton transformations associated with $T$ above defined and let $\left\{e_{1}, \ldots, e_{n}\right\}$ be a local orthonormal frame on $M$. Then, for all $Z \in \mathfrak{X}(M)$, the divergence of $P_{k}(T)$ are given recursively as

$$
\operatorname{div} P_{0}(T)=0,
$$

$$
\left\langle\operatorname{div} P_{k}(T), Z\right\rangle=-\left\langle T\left(\operatorname{div} P_{k-1}(T)\right), Z\right\rangle-\sum_{i=1}^{n} D\left(e_{i}, Z, P_{k-1}(T) e_{i}\right),
$$

or equivalently

$$
\left\langle\operatorname{div} P_{k}(T), Z\right\rangle=\sum_{j=1}^{k} \sum_{i=1}^{n}(-1)^{j} D\left(e_{i}, T^{j-1} Z, P_{k-j}(T) e_{i}\right) .
$$

Proof. Since $P_{0}(T)=I$, then $\operatorname{div} P_{0}(T)=0$. By the inductive definition of $P_{k}(T)$, we have

$$
\begin{aligned}
\nabla_{Z} P_{k}(T) Y & =\left\langle\nabla \sigma_{k}(T), Z\right\rangle Y-\nabla_{Z}\left(T \circ P_{k-1}(T)\right) Y \\
& =\left\langle\nabla \sigma_{k}(T), Z\right\rangle Y-\left(\nabla_{Z} T \circ P_{k-1}(T)\right) Y-\left(T \circ \nabla_{Z} P_{k-1}(T)\right) Y,
\end{aligned}
$$

so that

$$
\begin{aligned}
\operatorname{div} P_{k}(T) & =\sum_{i=1}^{n}\left(\nabla_{e_{i}} P_{k}(T)\right) e_{i} \\
& =\nabla \sigma_{k}(T)-\sum_{i=1}^{n}\left(\nabla_{e_{i}} T\right)\left(P_{k-1}(T) e_{i}\right)-\left(T \operatorname{div} P_{k-1}(T)\right) .
\end{aligned}
$$

Now, by using (2.10), we get

$$
\begin{aligned}
\left\langle\left(\nabla_{e_{i}} T\right)\left(P_{k-1}(T) e_{i}\right), Z\right\rangle & =\left\langle\left(\nabla_{e_{i}} T\right) Z, P_{k-1}(T) e_{i}\right\rangle \\
& =\left(\nabla_{e_{i}} T\right)\left(Z, P_{k-1}(T) e_{i}\right) \\
& =D\left(e_{i}, Z, P_{k-1}(T) e_{i}\right)+\nabla_{Z} T\left(e_{i}, P_{k-1}(T) e_{i}\right) \\
& =D\left(e_{i}, Z, P_{k-1}(T) e_{i}\right)+\left\langle\left(\nabla_{Z} T\right) e_{i}, P_{k-1}(T) e_{i}\right\rangle \\
& =D\left(e_{i}, Z, P_{k-1}(T) e_{i}\right)+\left\langle\left(P_{k-1}(T) \circ \nabla_{Z} T\right)\left(e_{i}\right), e_{i}\right\rangle .
\end{aligned}
$$

Therefore, letting $\rho=\sum_{i=1}^{n} D\left(e_{i}, Z, P_{k-1}(T) e_{i}\right)$, we deduce

$$
\begin{aligned}
\left\langle\operatorname{div} P_{k}(T), Z\right\rangle= & \left\langle\nabla \sigma_{k}(T), Z\right\rangle-\operatorname{tr}\left(P_{k-1}(T) \circ \nabla_{Z} T\right) \\
& -\left(T \operatorname{div} P_{k-1}(T)\right)-\rho .
\end{aligned}
$$


Now we just need to prove that

$$
\operatorname{tr}\left(P_{k-1}(T) \circ \nabla_{Z} T\right)=\left\langle\nabla \sigma_{k}(T), Z\right\rangle .
$$

We prove the above equation using a local orthonormal frame that diagonalizes $T$. We point out that such a frame does not always exist, since the multiplicity of the eigenvalues may change. Therefore, we will work in a subset $M_{T} \subset M$ consisting of points at which the multiplicity of the eigenvalues is locally constant. We recall that such subset is open and dense in $M$, and in every connected component of $M_{T}$ the eigenvalues form mutually smooth distinct eigenfunctions and, for such a function $\lambda$, the assignment $p \rightarrow V_{\lambda(p)}(p) \subset T_{p} M$ defines a smooth eigenspace distribution $V_{\lambda}$ of $T$ (consult [4], Paragraph 16.10). Therefore, for every $p \in M_{T}$ there exists a local orthonormal frame defined on a neighborhood of $p$ that diagonalizes $T$, that is,

$$
\left(\nabla_{Z} T\right) e_{i}=Z\left(\lambda_{i}\right) e_{i}+\sum_{j \neq i}\left(\lambda_{i}-\lambda_{j}\right) \omega_{i}^{j}(Z) e_{j}
$$

where we use the standard notation $\omega_{i}^{j}(Z)=\left\langle\nabla_{Z} e_{i}, e_{j}\right\rangle$. Using (2.8) we get

$$
\begin{aligned}
\operatorname{tr}\left(P_{k-1}(T) \circ \nabla_{Z} T\right) & =\sum_{i=1}^{n} \mu_{i, k-1} Z\left(\lambda_{i}\right) \\
& =\sum_{i=1}^{n} Z\left(\lambda_{i}\right) \sum_{i_{1}<\cdots<i_{k}, i_{j} \neq i} \lambda_{i_{1}} \cdots \lambda_{i_{k-1}} \\
& =Z\left(\sum_{i_{1}<\cdots<i_{k}} \lambda_{i_{1}}(T) \cdots \lambda_{i_{k}}(T)\right)=\left\langle\nabla \sigma_{k}(T), Z\right\rangle .
\end{aligned}
$$

This proves the statement on $M_{T}$, and by continuity on $M$. Substituting (2.14) into (2.13), we get (2.11). In order to arrive at (2.12) it suffices to use an inductive argument.

In particular we have the following.

Corollary 1. If $D=0$, then the Newton transformations are divergence free: $\operatorname{div} P_{k}(T)=0$ for each $k$.

2.3. Integral formulae. Let $(M, g, X, \lambda)$ be an almost Ricci soliton. Now take $T$ to be the Schouten tensor, that is, $T=A$. Note that $\nabla_{Y} P_{k}(A)$ is self-adjoint for all $Y \in \mathfrak{X}(M)$. A straightforward computation shows that

$$
\operatorname{div}\left(P_{k}(A) X\right)=\left\langle\operatorname{div} P_{k}(A), X\right\rangle+\sum_{i=1}^{n}\left\langle\nabla_{e_{i}} X, P_{k}(A) e_{i}\right\rangle
$$

where $\left\{e_{1}, \ldots, e_{n}\right\}$ is a local orthonormal frame. If we take such a local orthonormal frame that diagonalizes $A$, then by (2.8) we have

$$
\left\langle\nabla_{e_{i}} X, P_{k}(A) e_{i}\right\rangle=\mu_{i, k}\left\langle\nabla_{e_{i}} X, e_{i}\right\rangle=\left\langle\nabla_{P_{k}(A) e_{i}} X, e_{i}\right\rangle .
$$


By the almost Ricci soliton equation (1.1), we get

$$
\begin{aligned}
\left\langle\nabla_{P_{k}(A) e_{i}} X, e_{i}\right\rangle & =\lambda\left\langle P_{k}(A) e_{i}, e_{i}\right\rangle-\operatorname{Ric}\left(P_{k}(A) e_{i}, e_{i}\right) \\
& =\left(\lambda-\frac{R}{2(n-1)}\right)\left\langle P_{k}(A) e_{i}, e_{i}\right\rangle-\left\langle A P_{k}(A) e_{i}, e_{i}\right\rangle,
\end{aligned}
$$

hence, using equations (2.16) and (2.9), equation (2.15) becomes

$$
\begin{aligned}
\operatorname{div}\left(P_{k}(A) X\right) & =\left\langle\operatorname{div} P_{k}(A), X\right\rangle+\left(\lambda-\frac{R}{2(n-1)}\right) \operatorname{tr} P_{k}(A)-\operatorname{tr}\left(A P_{k}(A)\right) \\
& =\left\langle\operatorname{div} P_{k}(A), X\right\rangle+\left(\lambda-\frac{R}{2(n-1)}\right) c_{k} S_{k}(A)-c_{k} S_{k+1}(A) .
\end{aligned}
$$

Taking trace in (1.1) we get $R+\operatorname{div} X=n \lambda$. Since $S_{1}(A)=\frac{(n-2)}{2 n(n-1)} R$ we obtain

$$
\begin{aligned}
\operatorname{div}\left(P_{k}(A) X\right)= & \left\langle\operatorname{div} P_{k}(A), X\right\rangle+\left(S_{1}(A)+\frac{1}{n} \operatorname{div} X\right) c_{k} S_{k}(A) \\
& -c_{k} S_{k+1}(A)
\end{aligned}
$$

When $M$ is a compact manifold and $h$ is the function given by the Hodge-de Rham decomposition theorem it is easy to see that identity (2.17) becomes

$$
\begin{aligned}
\operatorname{div}\left(P_{k}(A) X\right)= & \left\langle\operatorname{div} P_{k}(A), X\right\rangle+\left(S_{1}(A)+\frac{1}{n} \Delta h\right) c_{k} S_{k}(A) \\
& -c_{k} S_{k+1}(A) .
\end{aligned}
$$

Lemma 2. Let $(M, g, X, \lambda)$ be a compact oriented almost Ricci soliton. For each $k$, the following integral formula holds:

$$
\begin{aligned}
& \int_{M}\left\langle\operatorname{div} P_{k}(A), X\right\rangle d V_{g} \\
& \quad+c_{k} \int_{M}\left(\left(S_{1}(A)+\frac{1}{n} \Delta h\right) S_{k}(A)-S_{k+1}(A)\right) d V_{g}=0 .
\end{aligned}
$$

Note that when the Cotton tensor vanishes Corollary 1 implies that

$$
\int_{M}\left\langle\operatorname{div} P_{k}(A), X\right\rangle d V_{g}=0
$$

Therefore, we obtain the next corollary.

Corollary 2. Let $(M, g, X, \lambda)$ be a compact oriented almost Ricci soliton such that the Cotton tensor vanishes. Then,

$$
\int_{M}\left(\left(S_{1}(A)+\frac{1}{n} \Delta h\right) S_{k}(A)-S_{k+1}(A)\right) d V_{g}=0
$$




\section{Proof of the main results}

REMARK 1. Before presenting the proofs of the results, we recall that the symmetric functions satisfy Newton's inequalities:

$$
S_{k}(A) S_{k+2}(A) \leq S_{k+1}^{2}(A) \text { for } 0 \leq k<n-1,
$$

which is a generalized Cauchy-Schwarz inequality. Moreover, if equality occurs for $k=0$ or $1 \leq k<n$ with $S_{k+2}(A) \neq 0$, then $\lambda_{1}(A)=\lambda_{2}(A)=\cdots=$ $\lambda_{n}(A)$. As an application, provided that $\lambda_{k}(A)>0$ for $1 \leq k \leq n$, we obtain Gårding's inequalities

$$
S_{1} \geq S_{2}^{\frac{1}{2}} \geq S_{3}^{\frac{1}{3}} \geq \cdots \geq S_{n}^{\frac{1}{n}} .
$$

Here equality holds, for some $1 \leq k<n$, if and only if $\lambda_{1}(A)=\lambda_{2}(A)=\cdots=$ $\lambda_{n}(A)$. Note that (3.2) implies that $S_{k}^{\frac{k+1}{k}} \geq S_{k+1}$ for $1 \leq k<n$. For a proof see for instance [11], Theorem 51, p. 52 or Proposition 1 in [7].

\subsection{Proof of Theorem 1.}

Proof. In item (1), we suppose that $S_{2}(A)$ is constant and positive. Thereby, choosing $k=2$ in (2.21) we obtain

$$
\int_{M}\left(\left(S_{1}(A)+\frac{1}{n} \Delta h\right) S_{2}(A)-S_{3}(A)\right) d V_{g}=0 .
$$

Since $S_{2}(A)$ is constant we deduce

$$
\int_{M}\left(S_{2}(A) S_{1}(A)-S_{3}(A)\right) d V_{g}=0
$$

On the other hand,

$$
S_{1}^{2}(A)-S_{2}(A) \geq 0,
$$

by Newton's inequality (3.1). Moreover, equality in (3.5) holds if and only if $\lambda_{1}(A)=\cdots=\lambda_{n}(A)$, which means that $A$ is umbilical (a multiple of $g$ ). In this case, it is easy to check that

$$
A=\frac{(n-2)}{2 n(n-1)} R g .
$$

We know from (3.5) that $S_{1}^{2}(A) \geq S_{2}(A)>0$, then $S_{1}(A)$ does not vanish, this means that either $S_{1}(A)<0$ or $S_{1}(A)>0$. Now we prove that $S_{2}(A) S_{1}(A)-$ $S_{3}(A)$ is positive or negative, according to the sign of $S_{1}(A)$.

Indeed, from $(3.1)$ we get $S_{2}^{2}(A)-S_{1}(A) S_{3}(A) \geq 0$. Supposing $S_{1}(A)>0$ we obtain

$$
\begin{aligned}
S_{2}(A) S_{1}(A)-S_{3}(A) & \geq S_{2}(A) S_{1}(A)-\frac{S_{2}^{2}(A)}{S_{1}(A)} \\
& =\frac{S_{2}(A)}{S_{1}(A)}\left(S_{1}^{2}(A)-S_{2}(A)\right) \geq 0 .
\end{aligned}
$$


On the other hand, if $S_{1}(A)<0$ we have

$$
\begin{aligned}
S_{2}(A) S_{1}(A)-S_{3}(A) & \leq S_{2}(A) S_{1}(A)-\frac{S_{2}^{2}(A)}{S_{1}(A)} \\
& =\frac{S_{2}(A)}{S_{1}(A)}\left(S_{1}^{2}(A)-S_{2}(A)\right) \leq 0 .
\end{aligned}
$$

In both cases $S_{2}(A) S_{1}(A)-S_{3}(A)$ has a sign. Using this fact together with equation (3.4), we get $S_{2}(A) S_{1}(A)-S_{3}(A)=0$, and hence equality in (3.1), obtaining identity (3.6). Therefore $\left(M^{n}, g\right)$ is an Einstein manifold, and by equation (1.1) we have that $X$ is a conformal vector field on $M$. If $R \leq$ 0 , since every conformal vector field on a connected compact manifold with nonpositive scalar curvature is Killing (see, for instance, Theorem 6 in [14]), we contradict the assumption that $(M, g, X, \lambda)$ is a non-trivial almost Ricci soliton. Then, $R$ is constant and positive. Therefore, we are in position to make use of Corollary 1 in [2] to conclude that $\left(M^{n}, g\right)$ is isometric to a standard sphere $\mathbb{S}^{n}$, which concludes the proof of the first point of Theorem 1.

Proceeding, in item (2) we assume that $S_{k}(A)$ is nowhere zero on $M$ and $S_{k+1}(A)=c S_{k}(A)$, where $c \in \mathbb{R} \backslash\{0\}$, for some $k=1, \ldots, n-1$. Thus we can use Corollary 2 to infer

$$
\int_{M}\left(\left(S_{1}(A)+\frac{1}{n} \Delta h\right) c S_{k}(A)-c S_{k+1}(A)\right) d V_{g}=0
$$

and

$$
\int_{M}\left(\left(S_{1}(A)+\frac{1}{n} \Delta h\right) S_{k+1}(A)-S_{k+2}(A)\right) d V_{g}=0 .
$$

By hypothesis $S_{k+1}(A)=c S_{k}(A)$, whence using (3.10) and (3.9) we deduce

$$
\int_{M}\left(c S_{k+1}(A)-S_{k+2}(A)\right) d V_{g}=0 .
$$

Using once more that $S_{k+1}(A)=c S_{k}(A)$ we invoke inequality (3.1) to get $S_{k}(A)\left(c S_{k+1}(A)-S_{k+2}(A)\right) \geq 0$. We recall that $S_{k}(A)$ is nowhere zero on $M$ by hypothesis, and by connectedness it does not change sign on $M$. Hence, $c S_{k+1}(A)-S_{k+2}(A)$ also does not change sign on $M$. This fact together with equation (3.11) gives $c S_{k+1}(A)-S_{k+2}(A)=0$, which implies $S_{k+1}^{2}(A)-$ $S_{k}(A) S_{k+2}(A)=0$ and consequently $S_{k+2}(A) \neq 0$ on $M$. Then equality in the inequality (3.1) implies that $A=\frac{(n-2)}{2 n(n-1)} R g$ and $g$ is an Einstein metric on $M$ and we conclude the proof reasoning as in the previous case.

We suppose in item (3) that Ric $\geq \frac{R}{n} g$, with $R>0$, and $\int_{M} S_{k}(A) \Delta h \geq 0$ for some $2 \leq k \leq n-1$, hence $A \geq \frac{(n-2)}{2 n(n-1)} R g$, which implies that $A>0$. This allows us to use all inequalities presented in Remark 1. By identity (2.21), we 
have

$$
\int_{M}\left(\left(S_{1}(A)+\frac{1}{n} \Delta h\right) S_{k}(A)-S_{k+1}(A)\right) d V_{g}=0 .
$$

Taking into account that $\int_{M} S_{k}(A) \Delta h d V_{g} \geq 0$, we deduce from the last identity

$$
\int_{M}\left(S_{1}(A) S_{k}(A)-S_{k+1}(A)\right) d V_{g} \leq 0
$$

Next, we make use of (3.2) to arrive at

$$
\begin{aligned}
S_{1}(A) S_{k}(A)-S_{k+1}(A) & \geq S_{1}(A) S_{k}(A)-S_{k}(A)^{\frac{k+1}{k}} \\
& =S_{k}(A)\left(S_{1}(A)-S_{k}(A)^{\frac{1}{k}}\right) \geq 0 .
\end{aligned}
$$

Hence, using (3.13) and (3.14) we obtain $S_{1}(A) S_{k}(A)-S_{k+1}(A)=0$. Since $A>0$ and hence $S_{k}(A), S_{k+2}(A)>0$, using (3.14) again we obtain $S_{1}(A)=$ $S_{k}(A)^{\frac{1}{k}}$. By equality in (3.2) we get $A=\frac{(n-2)}{2 n(n-1)} R g$ and $g$ is an Einstein metric on $M$. Now, in order to complete the proof it suffices to use once more Corollary 1 in [2].

Finally, we assume in item (4) that $S_{k}(A)$ is constant for some $k=$ $2, \ldots, n-1$, and $A>0$. Hence, applying the same argument used to prove item (3) we conclude that $S_{1}(A)=S_{k}(A)^{\frac{1}{k}}>0$ and $S_{k+2}(A)>0$, which implies that $g$ is an Einstein metric on $M$. Since $S_{1}(A)=\frac{(n-2)}{2 n(n-1)} R$, we get $R>0$. The result follows by the same argument used to conclude item (3), completing the proof of the theorem.

Acknowledgment. The authors would like to thank the referee for valuable suggestions for the improvement of the paper.

\section{REFERENCES}

[1] A. Barros, R. Batista and E. Jr. Ribeiro, Rigidity of gradient almost Ricci solitons, Illinois J. Math. 56 (2012), 1267-1279. MR 3231482

[2] A. Barros, R. Batista and E. Jr. Ribeiro, Compact almost Ricci solitons with constant scalar curvature are gradient, Monatsh. Math. 174 (2014), no. 1, 29-39. MR 3190769

[3] A. Barros and E. Jr. Ribeiro, Some characterizations for compact almost Ricci solitons, Proc. Amer. Math. Soc. 140 (2012), 1033-1040. MR 2869087

[4] A. Besse, Einstein manifolds, Springer, New York, 2008. MR 2371700

[5] A. Brasil, E. Costa and E. Jr. Ribeiro, Hitchin-Thorpe inequality and Kaehler metrics for compact almost Ricci soliton, Ann. Mat. Pura Appl. (4) 193 (2014), 1851-1860. MR 3275265

[6] M. Brozos-Vázquez, E. García-Río and X. Valle-Regueiro, Half conformally flat gradient Ricci almost solitons, Proc. R. Soc. Lond. Ser. A Math. Phys. Eng. Sci. 472, 20160043 (2016). MR 3511627

[7] A. Caminha, On spacelike hypersurfaces of constant sectional curvature Lorentz manifolds, J. Geom. Phys. 56 (2006), no. 7, 1144-1174. MR 2234043

[8] H.-D. Cao, Recent progress on Ricci soliton, Adv. Lectures Math. 11 (2009), 1-38. MR 2648937 
[9] G. Catino, L. Cremaschi, Z. Djadli, C. Mantegazza and L. Mazzieri, The RicciBourguignon flow, Pacific J. Math. 287 (2017), 337-370. MR 3632891

[10] A. Ghosh, Certain contact metrics as Ricci almost solitons, Results Math. 65 (2014), 81-94. MR 3162430

[11] G. Hardy, J. E. Littlewood and G. Póyla, Inequalities, 2nd ed., Cambridge Mathematical Library, Cambridge, 1989. MR 0944909

[12] Z. Hu, H. Li and U. Simon, Schouten curvature functions on locally conformally flat Riemannian manifolds, J. Geom. 88 (2008), 75-100. MR 2398477

[13] G. Maschler, Almost soliton duality, Adv. Geom. 15 (2015), 159-166. MR 3334021

[14] M. Obata, Conformal transformations of compact Riemannian manifolds, Illinois J. Math. 6 (1962), 292-295. MR 0138059

[15] S. Pigola, M. Rigoli, M. Rimoldi and A. Setti, Ricci almost solitons, Ann. Sc. Norm. Super. Pisa Cl. Sci. (5) X (2011), 757-799. MR 2932893

[16] R. Sharma, Almost Ricci solitons and K-contact geometry, Monatsh. Math. 175 (2014), 621-628. MR 3273671

[17] F. Warner, Foundations of differentiable manifolds and Lie groups, Springer, New York, 1983. MR 0722297

A. Barros, Departamento de Matemática-UFC, 60455-760-Fortaleza-CE, Brazil E-mail address: abbarros@mat.ufc.br; URL: http://www.mat.ufc.br

I. Evangelista, Departamento de Matemática-UFPi, 64202-020-Parnaíba-Pi, BRAZIL

E-mail address: israelevangelista@ufpi.edu.br 